\title{
Closure of the Monte Carlo dynamical equations in the spherical Sherrington-Kirkpatrick model
}

\author{
L. L. Bonilla* and F. G. Padilla ${ }^{\dagger}$ \\ Departamento de Matemáticas, Universidad Carlos III, Butarque 15, Leganés 28911, Madrid, Spain
}

\author{
G. Parisi ${ }^{\ddagger}$ \\ Dipartimento di Fisica, Università di Roma I "La Sapienza,"' Istituto Nazionale di Fisica Nucleare Sezione di Roma I, \\ Piazzale Aldo Moro, Roma 00187, Italy \\ F. Ritort ${ }^{\S}$ \\ Departamento de Matemáticas, Universidad Carlos III, Butarque 15, Leganés 28911, Madrid, Spain
}

(Received 29 February 1996)

\begin{abstract}
We study the analytical solution of the Monte Carlo dynamics in the spherical Sherrington-Kirkpatrick model using the technique of the generating function. Explicit solutions for one-time observables (like the energy) and two-time observables (like the correlation and response function) are obtained. We show that the crucial quantity which governs the dynamics is the acceptance rate. At zero temperature, an adiabatic approximation reveals that the relaxational behavior of the model corresponds to that of a single harmonic oscillator with an effective renormalized mass. [S0163-1829(96)00129-4]
\end{abstract}

\section{INTRODUCTION}

The off-equilibrium features of glassy systems constitute a very interesting field of research. A great deal of attention has been paid to the study of the dynamics of exactly solvable models of disordered systems where much relevant information can be gathered even in the mean-field case. $\mathrm{Nu}$ merical simulations of short-ranged and long-ranged models reveal a similar nature of the off-equilibrium dynamics. ${ }^{1-4}$ The outstanding phenomenon which characterizes the offequilibrium regime is the presence of aging, which has been experimentally observed in a large class of physical systems (for instance, in spin glasses ${ }^{5}$ as well as in real glasses ${ }^{6}$ ). The distinctive feature of the aging phenomenon is that the response of the system to an external perturbation depends strongly on the time elapsed since the perturbation was applied. A phenomenological approach was proposed by Bouchaud $^{7}$ where the slow dynamics originates from the presence of a large number of traps in phase space with a very broad distribution of lifetimes.

Much interest has been also devoted to the study of exactly solvable mean-field spin-glass models where information can be obtained from the solution of the dynamical equations. Of particular interest is the study of disordered models where replica symmetry is broken in the lowtemperature phase. In this case, several results have been obtained in the study of $p$-spin models ${ }^{8-10}$ as well as in the study of a particle in a random potential. ${ }^{11-13}$ From these studies a very interesting connection ${ }^{14}$ between mean-field glassy dynamical equations and the mode coupling theory of glasses ${ }^{15,16}$ has emerged. In this theoretical framework one derives a closed system of integro-differential equations for the two-time correlation and response functions. Whereas all the information about the dynamics is contained in this system of equations, it is very difficult to ascertain the long-time evolution of one-time observables like the energy or the magnetization. Despite several advances in this direction (see, for instance, the time series expansion carried out by Franz, Marinari, and Parisi in case of the $p=3$ spherical spin-glass model ${ }^{17}$ ) this is still an open problem. Recently a completely different approach to the dynamics of glassy systems has been proposed by Coolen and Sherrington. ${ }^{18}$ They introduce a closure assumption (the equipartitioning hypothesis) which yields evolution equations for the one-time observables. In comparison with the results of simulations they obtain fairly good results in the short-time regime. However, it is still unclear whether the equipartitioning approximation is applicable for long times and how to proceed where this is the case. ${ }^{19,20}$

On the issue of spin-glass dynamics, most of the existing works study Langevin equations. Meanwhile Monte Carlo dynamics has received less attention notwithstanding its great importance in numerical simulations. ${ }^{22}$ In particular, given that a major part of the numerical work in spin glasses uses the Monte Carlo algorithm, we think that it is important to study the Monte Carlo dynamics in itself. Notice that Monte Carlo dynamics introduces the concept of an acceptance rate which has no meaning for the Langevin dynamics. Interestingly, we shall see that the nature of the offequilibrium regime strongly depends on the behavior of the acceptance rate. To shed some light on these issues we have considered the spherical Sherrington-Kirkpatrick (SK) model. ${ }^{21}$ While this model lacks replica symmetry breaking in the low-temperature phase, it can be analytically solved.

In another paper (hereafter referred as I) we introduced a method to solve the Monte Carlo dynamics of the spherical SK model. ${ }^{23}$ We derived generalized dynamical equations for one-time quantities and a closed integro-differential problem for their generating function. The same method can be successfully applied to models with Langevin dynamics. The purpose of this work is to present the method of the generating function, give a detailed account of the computations announced in I, and discuss some other topics such as the properties of two-time correlation and response functions 
and the long-time behavior of dynamical quantities.

The paper is organized as follows. In Sec. II we introduce the spherical SK model and explain how to solve its Langevin dynamics by the method of the generating function. While the results of this section are well known, ${ }^{24,25}$ their derivation by means of the generating function serves as a clear introduction of this method. Section III contains the main result of this paper, i.e., the solution of the corresponding equations for the Monte Carlo dynamics. Section IV analyzes the long-time behavior of dynamical quantities at finite and zero temperature and the conditions under which the results of the Langevin dynamics are recovered. Section V contains our conclusions while the Appendixes are devoted to different technical matters.

\section{LANGEVIN APPROACH: A REMINDER}

The spherical Sherrington-Kirkpatrick spin-glass model is defined by the Hamiltonian

$$
\mathcal{H}\{\sigma\}=-\sum_{i<j} J_{i j} \sigma_{i} \sigma_{j}
$$

where the indices $i, j$ run from 1 to $N$ ( $N$ is the number of sites) and the spins $\sigma_{i}$ satisfy the spherical global constraint

$$
\sum_{i=1}^{N} \sigma_{i}^{2}=N
$$

The interactions $J_{i j}$ are Gaussian distributed with zero mean and $1 / N$ variance. The statics of this model reveals the existence of a thermodynamic second-order phase transition at $T=1$ (Ref. 21 ) with a replica-symmetric low-temperature phase.

We will revisit the Langevin dynamics of the spherical SK model in order to introduce the technique of the generating function. The Langevin equation is

$$
\frac{\partial \sigma_{i}}{\partial t}=-\frac{\partial \mathcal{H}}{\partial \sigma_{i}}+\mu(t) \sigma_{i}+\eta_{i}(t)
$$

with $\eta_{i}(t)$ a Gaussian white noise, $\overline{\eta_{i}(t) \eta_{j}\left(t^{\prime}\right)}=2 T \delta_{i, j} \delta\left(t-t^{\prime}\right)(\bar{\cdots}$ stands for average over the noise); $\mu(t)$ is a time-dependent Lagrange multiplier which ensures that the spherical constraint, Eq. (2), is satisfied at all times, and $\mathcal{H}$ is the Hamiltonian defined by Eq. (1). We can rewrite the previous equation as a single mode problem,

$$
\frac{\partial \sigma_{\lambda}}{\partial t}=\lambda \sigma_{\lambda}+\mu(t) \sigma_{\lambda}+\eta_{\lambda}(t)
$$

Here $\sigma_{\lambda}$ and $\eta_{\lambda}$ are the projections of the configuration $\left\{\sigma_{i}\right\}$ and the original noise $\left\{\eta_{i}\right\}$ on the basis of eigenvectors which diagonalize the interaction matrix $J_{i j}$. The $\left\{\sigma_{\lambda}\right\}$ satisfy the spherical constraint $\Sigma_{\lambda=1}^{N} \sigma_{\lambda}^{2}=N$. The transformation matrix which diagonalizes the $J_{i j}$ is an orthogonal matrix. Hence, the components $\eta_{\lambda}$ generate a white noise of the type

$$
\overline{\eta_{\lambda}(t) \eta_{\lambda^{\prime}}\left(t^{\prime}\right)}=2 T \delta_{\lambda, \lambda^{\prime}} \delta\left(t-t^{\prime}\right)
$$

The eigenvalues $\lambda$ of a random Gaussian symmetric matrix are distributed according to the Wigner semicircular law $w(\lambda),^{27}$

$$
w(\lambda)=\frac{\sqrt{4-\lambda^{2}}}{2 \pi}
$$

\section{A. Generating function for the one-time quantities}

Our purpose is to describe the time evolution of certain one-time functions of the solutions of Eq. (4). We define the set of moments

$$
h_{k}=\frac{1}{N} \sum_{(i, j)} \overline{\sigma_{i}\left(J^{k}\right)_{i j} \sigma_{j}}=\frac{1}{N} \sum_{\lambda} \lambda^{k} \overline{\sigma_{\lambda}^{2}} .
$$

Notice that $h_{0}=1$ (spherical constraint) and $h_{1}=-2 E$ where $E$ is the energy. Using the result $\lim _{t \rightarrow t^{\prime}} \overline{\eta_{\lambda}\left(t^{\prime}\right) \sigma_{\lambda}(t)}=2 T$ we get the equation

$$
\frac{\partial h_{k}}{\partial t}=2 h_{k+1}+2 \mu h_{k}+2 T\left\langle\left\langle\lambda^{k}\right\rangle\right\rangle
$$

where

$$
\langle\langle f(\lambda)\rangle\rangle=\int_{-2}^{2} f(\lambda) w(\lambda) d \lambda
$$

and $w(\lambda)$ is given by Eq. (6). In particular, Eq. (8) for $k=0$ gives the Lagrange multiplier as a function of the energy and temperature, $\mu=2 E-T$. In order to close these equations we define the generating function

$$
g(x, t)=\frac{1}{N} \sum_{(i, j)} \sigma_{i}\left(e^{x J}\right)_{i j} \sigma_{j}=\frac{1}{N} \sum_{\lambda} e^{\lambda x} \sigma_{\lambda}^{2}(t)=\sum_{k=0}^{\infty} \frac{x^{k}}{k !} h_{k}(t) .
$$

This function yields all the moments $h_{k}=\left(\partial^{k} g(x, t) /\right.$ $\left.\partial x^{k}\right)_{x=0}$.

We now want to formulate a problem for $g(x, t)$ (equation, initial, boundary, and subsidiary conditions) which has a unique solution. Then we will either be able to solve it (and thus determine the $h_{k}$ 's exactly) or to infer the long-time behavior of the moments. By using Eqs. (7), (8), and (10), we find that $g(x, t)$ satisfies the differential equation

$$
\begin{gathered}
\frac{\partial g(x, t)}{\partial t}=2 \frac{\partial g(x, t)}{\partial x}+2 \mu(t) g(x, t)+2 T\langle\langle\exp (x \lambda)\rangle\rangle, \\
\mu(t)=-\frac{\partial g}{\partial x}(0, t)-T .
\end{gathered}
$$

These equations have to be solved with the boundary condition $g(0, t)=1$ (the spherical constraint) and an appropriate initial condition $g(x, 0)=g_{0}(x)$ [defined by the initial configuration $\left.\left\{\sigma_{\lambda}(t=0)\right\}\right]$. Besides taking the continuum limit in the definition (10), we see that

$$
g(x, t)=\int_{-2}^{2} d \lambda w(\lambda) e^{x \lambda} \hat{g}(\lambda, t)
$$

where the spectral transform $\hat{g}(\lambda, t) \geqslant 0$. The latter condition is kept by the dynamics if it holds initially. However, its use 
is crucial to distinguish the physically meaningful stationary solutions from spurious ones. We can solve (11) by the method of characteristics assuming that $\mu(t)$ is given. The result is

$$
\begin{aligned}
g(x, t)= & g_{0}(x+2 t) \exp \left(2 \int_{0}^{t} \mu\left(t^{\prime}\right) d t^{\prime}\right) \\
& +2 T \int_{0}^{t} d t^{\prime}\left\langle\left\langle e^{\left[x+2\left(t-t^{\prime}\right)\right] \lambda}\right\rangle\right\rangle \exp \left(2 \int_{t^{\prime}}^{t} \mu\left(t^{\prime \prime}\right) d t^{\prime \prime}\right) .
\end{aligned}
$$

Inserting (14) in the spherical constraint $g(0, t)=1$, it is possible to derive an integral equation which can be solved by means of the Laplace transform. ${ }^{25}$

It is easy to check that (15) is a stationary solution of Eq. (11) for $T>T_{c}=1$,

$$
g^{\mathrm{eq}}(x)=-\left\langle\left\langle\frac{e^{x \lambda}}{\beta\left(\lambda+\mu^{\mathrm{eq}}\right)}\right\rangle\right\rangle .
$$

In this case the moments $h_{k}$ can be easily computed, ${ }^{21}$

$$
h_{k}^{\mathrm{eq}}=-\left\langle\left\langle\frac{\lambda^{k}}{\beta\left(\lambda+\mu^{\mathrm{eq}}\right)}\right\rangle\right\rangle,
$$

and the Lagrange multiplier $\mu^{\text {eq }}$ is given by

$$
1=-\left\langle\left\langle\frac{1}{\beta\left(\lambda+\mu^{\mathrm{eq}}\right)}\right\rangle\right\rangle \text {. }
$$

For $T \leqslant 1, \mu^{\mathrm{eq}}=-2$, the stationary solution is given by

$$
g^{\mathrm{eq}}(x)=\left(1-\frac{1}{\beta}\right) e^{2 x}-\left\langle\left\langle\frac{e^{x \lambda}}{\beta(\lambda-2)}\right\rangle\right\rangle,
$$

and the moments $h_{k}$ are

$$
h_{k}^{\mathrm{eq}}=\left(1-\frac{1}{\beta}\right) 2^{k}-\left\langle\left\langle\frac{\lambda^{k}}{\beta(\lambda-2)}\right\rangle\right\rangle .
$$

Substituting Eqs. (15) and (18) in Eq. (11) one can easily check that the right-hand side (RHS) of Eq. (11) is identically zero.

\section{B. Correlation and response functions}

In order to find closed expressions for the correlation function we define the set of two-time moments,

$$
C_{k}\left(t^{\prime}, t\right)=\frac{1}{N} \sum_{(i, j)} \overline{\sigma_{i}\left(t^{\prime}\right)\left(J^{k}\right)_{i j} \sigma_{j}(t)}=\frac{1}{N} \sum_{\lambda} \lambda^{k} \overline{\sigma_{\lambda}\left(t^{\prime}\right) \sigma_{\lambda}(t)} .
$$

Note that in this notation the usual two-time correlation function is given by $C_{0}\left(t^{\prime}, t\right)$. The equation of motion for the $C_{k}\left(t^{\prime}, t\right)$ reads

$$
\frac{\partial C_{k}\left(t^{\prime}, t\right)}{\partial t}=C_{k+1}\left(t^{\prime}, t\right)+\mu(t) C_{k}\left(t^{\prime}, t\right),
$$

where we have used the result $\overline{\eta_{\lambda}\left(t^{\prime}\right) \sigma_{\lambda}(t)}=0$ for $t^{\prime}<t$. The previous equation has to be solved with the initial con- dition $C_{k}\left(t^{\prime}, t^{\prime}\right)=h_{k}\left(t^{\prime}\right)$ where the $h_{k}(t)$ are the one-time moments previously obtained from the generating function $g(x, t)$.

We define the generating function

$$
\begin{aligned}
K\left(x, t^{\prime}, t\right) & =\frac{1}{N} \sum_{(i, j)} \overline{\sigma_{i}\left(t^{\prime}\right)\left(e^{x J}\right)_{i j} \sigma_{j}(t)}=\frac{1}{N} \sum_{\lambda} e^{\lambda x} \overline{\sigma_{\lambda}\left(t^{\prime}\right) \sigma_{\lambda}(t)} \\
& =\sum_{k=0}^{\infty} \frac{x^{k}}{k !} C_{k}\left(t^{\prime}, t\right) .
\end{aligned}
$$

The generating function $K\left(x, t^{\prime}, t\right)$ yields the generalized two-time moments $C_{k}\left(t^{\prime}, t\right)=\left(\partial^{k} K\left(x, t^{\prime}, t\right) / \partial x^{k}\right)_{x=0}$ and satisfies the homogeneous partial differential equation

$$
\frac{\partial K\left(x, t^{\prime}, t\right)}{\partial t}=\frac{\partial K\left(x, t^{\prime}, t\right)}{\partial x}+\mu(t) K\left(x, t^{\prime}, t\right),
$$

with the initial condition $K\left(x, t^{\prime}, t^{\prime}\right)=g\left(x, t^{\prime}\right)$ and $g\left(x, t^{\prime}\right)$ is given in Eq. (14).

A similar method is applied to the response function. We define the set of two-time moments

$$
G_{k}\left(t^{\prime}, t\right)=\frac{1}{N} \sum_{(i, j)}\left(J^{k}\right)_{i j} \frac{\overline{\partial \sigma_{j}(t)}}{\partial \eta_{i}\left(t^{\prime}\right)}=\frac{1}{N} \sum_{\lambda} \lambda^{k} \frac{\overline{\partial \sigma_{\lambda}(t)}}{\partial \eta_{\lambda}\left(t^{\prime}\right)},
$$

where $t^{\prime}<t$. In this notation the usual response function is given by $G_{0}\left(t^{\prime}, t\right)$. We construct the generating function

$$
\begin{aligned}
\Gamma\left(x, t^{\prime}, t\right)=\frac{1}{N} \sum_{(i, j)}\left(e^{x J}\right)_{i j} \frac{\overline{\partial \sigma_{j}(t)}}{\partial \eta_{i}\left(t^{\prime}\right)} & =\frac{1}{N} \sum_{\lambda} e^{\lambda x} \frac{\overline{\partial \sigma_{\lambda}(t)}}{\eta_{\lambda}\left(t^{\prime}\right)} \\
& =\sum_{k=0}^{\infty} \frac{x^{k}}{k !} G_{k}\left(t^{\prime}, t\right) .
\end{aligned}
$$

The generating function $\Gamma\left(x, t^{\prime}, t\right)$ yields the generalized two-time moments $G_{k}\left(t^{\prime}, t\right)=\left(\partial^{k} \Gamma\left(x, t^{\prime}, t\right) / \partial x^{k}\right)_{x=0}$. With the usual regularization of the response function at equal times the dynamical equation for the $\Gamma\left(x, t^{\prime}, t\right)$ reads

$$
\begin{aligned}
\frac{\partial \Gamma\left(x, t^{\prime}, t\right)}{\partial t}= & \frac{\partial \Gamma\left(x, t^{\prime}, t\right)}{\partial x}+\mu(t) \Gamma\left(x, t^{\prime}, t\right) \\
& +\delta\left(t-t^{\prime}\right)\langle\langle\exp (x \lambda)\rangle\rangle .
\end{aligned}
$$

To solve this equation we need to impose the causality condition $\Gamma\left(x, t^{\prime}, t\right)=0$ if $t<t^{\prime}$. The solution to Eqs. (23) and (26) can be easily found. One gets the results

$$
K\left(x, t^{\prime}, t\right)=g\left(x+t-t^{\prime}, t^{\prime}\right) \exp \int_{t^{\prime}}^{t} \mu\left(t^{\prime \prime}\right) d t^{\prime \prime},
$$

$$
\Gamma\left(x, t^{\prime}, t\right)=\left\langle\left\langle e^{\left(x+t-t^{\prime}\right) \lambda}\right\rangle\right\rangle \exp \left(\int_{t^{\prime}}^{t} \mu\left(t^{\prime \prime}\right) d t^{\prime \prime}\right) \theta\left(t-t^{\prime}\right) .
$$

From these generating functions we can extract the usual two-time correlation and response functions $C_{0}\left(t^{\prime}, t\right)=K\left(0, t^{\prime}, t\right)$ and $G_{0}\left(t^{\prime}, t\right)=\Gamma\left(0, t^{\prime}, t\right)$. At equilibrium we can substitute the solution (15) in (26), obtaining 


$$
\begin{gathered}
K\left(x, t^{\prime}, t\right)=K^{\mathrm{eq}}\left(x, t-t^{\prime}\right)=g^{\mathrm{eq}}\left(x+t-t^{\prime}\right) e^{\mu^{\mathrm{eq}}\left(t-t^{\prime}\right)}, \\
\Gamma\left(x, t^{\prime}, t\right)=\Gamma^{\mathrm{eq}}\left(x, t-t^{\prime}\right)=\left\langle\left\langle e^{\left(x+t-t^{\prime}\right) \lambda}\right\rangle\right\rangle e^{\mu^{\mathrm{eq}}\left(t-t^{\prime}\right)} \theta\left(t-t^{\prime}\right) .
\end{gathered}
$$

Both functions are translationally invariant (i.e., depend only on the difference of times) and the fluctuation dissipation theorem for the generating functions also holds,

$$
\Gamma^{\mathrm{eq}}\left(x, t-t^{\prime}\right)=\beta \frac{\partial K^{\mathrm{eq}}\left(x, t-t^{\prime}\right)}{\partial t^{\prime}} .
$$

The off-equilibrium behavior of the quantities $C_{0}\left(t^{\prime}, t\right), G_{0}\left(t^{\prime}, t\right)$ has been already studied in the literature (see Refs. 24,25).

\section{MONTE CARLO APPROACH}

We consider the Monte Carlo (MC) dynamics with the Metropolis algorithm. The idea behind the MC approach is to postulate a dynamics in which a new configuration is proposed and accepted with a certain probability. The dynamics is ergodic at finite temperature and satisfies detailed balance. Following I we will consider a particularly simple motion which makes the dynamics exactly soluble: Take the configuration $\left\{\sigma_{i}\right\}$ at time $t$ and perform a small random rotation from that configuration to a new one $\left\{\tau_{i}\right\}$ where

$$
\tau_{i}=\sigma_{i}+\frac{r_{i}}{\sqrt{N}}
$$

and the $r_{i}$ are random numbers extracted from a Gaussian distribution $p(r)$ of finite variance $\rho$,

$$
p\left(r_{i}\right)=\frac{1}{\sqrt{2 \pi \rho^{2}}} \exp \left(-\frac{r_{i}^{2}}{2 \rho^{2}}\right) .
$$

Let us denote by $\Delta E$ the change of energy $\Delta E=E\{\tau\}-E\{\sigma\}$. According to the Metropolis algorithm we accept the new configuration with probability 1 if $\Delta E<0$ and with probability $\exp (-\beta \Delta E)$ if $\Delta E>0$, where $\beta=1 / T$ is the inverse of the temperature $T$.

\section{A. Probability distribution $P(\Delta E)$}

As before in the Langevin formulation, we want to obtain one- and two-time moments. It is useful to work in the basis for which the interaction matrix $J_{i j}$ is diagonal. The energy then reads

$$
E\left\{\sigma_{\lambda}\right\}=-\frac{1}{2} \sum_{\lambda} \lambda \sigma_{\lambda}^{2},
$$

where the $\sigma_{\lambda}$ are distributed according to Eq. (6). In this basis, the spin configuration still corresponds to a small random rotation; hence $\sigma_{\lambda} \rightarrow \sigma_{\lambda}+r_{\lambda} / \sqrt{N}$ where the new random numbers $r_{\lambda}$ are extracted from the same Gaussian distribution, Eq. (31).

The basic object we want to compute is the probability $P(\Delta E)$ of having a given variation $\Delta E$ of the energy $E$. This is a quantity which gives the average number of accepted changes. The variation $\Delta E^{*}$ of the energy $E$ in a Monte Carlo step is

$$
\Delta E^{*}=-\frac{1}{\sqrt{N}} \sum_{\lambda} \lambda \sigma_{\lambda} r_{\lambda}-\frac{1}{2 N} \sum_{\lambda} \lambda r_{\lambda}^{2},
$$

while the quantity $h_{0}=(1 / N) \Sigma_{\lambda} \sigma_{\lambda}^{2}$ is changed by the amount

$$
\Delta h_{0}^{*}=\frac{2}{\sqrt{N}} \sum_{\lambda} \sigma_{\lambda} r_{\lambda}+\frac{1}{N} \sum_{\lambda} r_{\lambda}^{2} .
$$

The probability $P(\Delta E)$ of having a change of energy is

$$
P(\Delta E)=\int \delta\left(\Delta E-\Delta E^{*}\right) \delta\left(\Delta h_{0}^{*}\right) \prod_{\lambda}\left[p\left(r_{\lambda}\right) d r_{\lambda}\right]
$$

where the last $\delta$ function in the integrand accounts for the spherical constraint and the variation $\Delta E^{*}$ is given in Eq. (33).

Using the integral representation for the $\delta$ function,

$$
\delta(x)=\frac{1}{2 \pi} \int_{-\infty}^{\infty} e^{i \alpha x} d \alpha
$$

and substituting in (35) we get

$$
\begin{aligned}
P(\Delta E)= & \int d \mu d \eta \exp \left[i \mu \Delta E-\frac{\rho^{2}}{2 N} \sum_{\lambda} \frac{\sigma_{\lambda}^{2} \gamma_{\lambda}^{2}}{\left(1-i \gamma_{\lambda}^{2} \rho^{2} / N\right)}\right. \\
& \left.-\frac{1}{2} \sum_{\lambda} \ln \left(1-\frac{i \gamma_{\lambda}^{2} \rho^{2}}{N}\right)\right]
\end{aligned}
$$

where $\gamma_{\lambda}=\mu \lambda+2 \eta$. After expanding the logarithm and retaining the first $1 / N$ correction we get (after some manipulations)

$$
P(\Delta E)=\frac{1}{\sqrt{2 \pi \rho^{2} B_{1}}} \exp \left(-\frac{\left(\Delta E+\rho^{2} E\right)^{2}}{2 \rho^{2} B_{1}}\right),
$$

with

$$
B_{1}=h_{2}-4 E^{2}\left(h_{0}=1\right), \quad h_{2}=\frac{1}{N} \sum_{\lambda} \lambda^{2} \sigma_{\lambda}^{2} .
$$

The equation for the energy is obtained by considering the average variation of energy in a MC step. In this case one MC step corresponds to $N$ elementary moves. In the thermodynamic limit we can write the continuous equations

$$
\begin{aligned}
\frac{\partial E}{\partial t}= & \overline{\Delta E}=\int_{-\infty}^{0}(\Delta) E P(\Delta E) d(\Delta E) \\
& +\int_{0}^{\infty}(\Delta E) \exp (-\beta \Delta E) P(\Delta E) d(\Delta E) .
\end{aligned}
$$

We can check that equilibrium is a stationary solution of the Monte Carlo dynamics. Using standard static calculations $^{28,21}$ one can show that in equilibrium $B_{1}=h_{2}-4 E^{2}=-2 E T$. In this case a straightforward com- 
putation shows that detailed balance is fulfilled. This means that, for a given value of $\Delta E$, the first integral appearing in the RHS of Eq. (40) cancels the contribution of the second integral in the RHS of Eq. (40) for the same value of $\Delta E$. In other words,

$$
P(-\Delta E)=\exp (-\beta \Delta E) P(\Delta E) .
$$

The equation for the energy reads

$$
\frac{\partial E}{\partial t}=-\frac{a(t)}{2} h_{2}(t)+b(t) E(t),
$$

where the coefficients $a(t)$ and $b(t)$ are given by

$$
\begin{gathered}
a(t)=\rho^{2} \beta e^{\left(\rho^{2} \beta / 2\right)\left(\beta B_{1}+2 E\right)} \operatorname{Erf}\left(\rho \beta \sqrt{\frac{2}{B_{1}}}-\alpha\right), \\
b(t)=-\frac{1}{2}\left[\rho^{2} \operatorname{Erf}(\alpha)+(4 E-T) a(t)\right],
\end{gathered}
$$

and $\operatorname{Erf}(x)$ is the complementary error function defined as $\operatorname{Erf}(x)=(2 / \sqrt{\pi}) \int_{x}^{\infty} d x \exp \left(-x^{2}\right)$ and the parameter $\alpha$ is given by

$$
\alpha=-\frac{\rho E}{\sqrt{2 B_{1}}} .
$$

Note that the quantities $E, B_{1}$, and $\alpha$ depend on time. Also one can compute the acceptance rate as a function of time, which is the probability of accepting a certain change of the configuration

$A(t)=\int_{-\infty}^{0} P(\Delta E) d(\Delta E)+\int_{0}^{\infty} \exp (-\beta \Delta E) P(\Delta E) d(\Delta E)$.

A straightforward computation shows

$$
A(t)=\frac{\operatorname{Erf}(\alpha)}{2}+\frac{1}{2} e^{\left(\rho^{2} \beta / 2\right)\left(\beta B_{1}+2 E\right)} \operatorname{Erf}\left(\rho \beta \sqrt{\frac{2}{B_{1}}}-\alpha\right) \text {. }
$$

In order to obtain the time evolution of the acceptance rate and to solve Eq. (42) of the time evolution of the energy we need to know the energy $E$ and $h_{2}$ at time $t$. Unfortunately, one can see that the time evolution equation for $h_{2}(t)$ involves $h_{3}(t)$ and so on. This hierarchy of moments can be closed introducing a generating function ${ }^{23}$ as has been done in the Langevin dynamics. This is the purpose of the next section.

\section{B. Generating function for the one-time quantities}

To close the equations of motion we consider the set of moments defined in Eq. (7). The basic object to compute is the joint probability distribution $P\left(\Delta h_{k}, \Delta E\right)$. This quantity can be written as

$$
\begin{aligned}
P\left(\Delta h_{k}, \Delta E\right)= & \int \delta\left(\Delta h_{k}-\Delta h_{k}^{*}\right) \delta(\Delta E \\
& \left.-\Delta E^{*}\right) \delta\left(\Delta h_{0}\right) \prod_{\lambda}\left[p\left(r_{\lambda}\right) d r_{\lambda}\right],
\end{aligned}
$$

where the last $\delta$ function in the integrand accounts for the spherical constraint and the variation $\Delta E^{*}$ is given in Eq. (33) while the variation $\Delta h_{k}^{*}$ is given by

$$
\Delta h_{k}^{*}=\frac{2}{\sqrt{N}} \sum_{\lambda} \lambda^{k} \sigma_{\lambda} r_{\lambda}+\frac{1}{N} \sum_{\lambda} \lambda^{k} r_{\lambda}^{2}
$$

Following the same technical steps as in the derivation of $P(\Delta E)$ we obtain the result

$$
P\left(\Delta h_{k}, \Delta E\right)=P(\Delta E) P\left(\Delta h_{k} \mid \Delta E\right)
$$

where $P(\Delta E)$ is the probability distribution, Eq. (38), and $P\left(\Delta h_{k} \mid \Delta E\right)$ is the conditional probability of $\Delta h_{k}$ given $\Delta E$. The final expression for the conditional probability is

$$
P\left(\Delta h_{k} \mid \Delta E\right)=\frac{1}{\sqrt{8 \pi \rho^{2}\left[C_{k}-\left(B_{k}^{2} / B_{1}\right)\right]}} \exp \left(-\frac{\left[\Delta h_{k}+\rho^{2}\left(h_{k}-\left\langle\left\langle\lambda^{k}\right\rangle\right\rangle\right)+2\left(B_{k} / B_{1}\right)\left(\Delta E+\rho^{2} E\right)\right]^{2}}{8 \rho^{2}\left(C_{k}-B_{k}^{2} / B_{1}\right)}\right)
$$

with $C_{k}=h_{2 k}-h_{k}^{2}, B_{k}=h_{k+1}+2 E h_{k}\left(h_{0}=1, h_{1}=-2 E\right)$, and the average $\langle\langle\cdots\rangle\rangle$ has been previously defined in Eq. (9).

In order to obtain the dynamical evolution of the moments $h_{k}$ we have to compute its average variation in a MC step over the accepted changes of configuration. In the thermodynamic limit we can write the continuous equations

$$
\begin{aligned}
\frac{\partial h_{k}}{\partial t}= & \overline{\Delta h_{k}}=\int_{-\infty}^{\infty} d\left(\Delta h_{k}\right) \Delta h_{k}\left(\int_{-\infty}^{0} d(\Delta E) P\left(\Delta h_{k}, \Delta E\right)\right. \\
& \left.+\int_{0}^{\infty} d(\Delta E) \exp (-\beta \Delta E) P\left(\Delta h_{k}, \Delta E\right)\right)
\end{aligned}
$$

The solution for a general integral of the previous type is shown in Appendix A. The following result is obtained:

$$
\frac{\partial h_{k}(t)}{\partial t}=a(t) h_{k+1}(t)+b(t) h_{k}(t)+c_{k}(t)
$$

where the time-dependent quantities $a(t)$ and $b(t)$ are given in Eq. (43) and the coefficients $c_{k}(t)$ are defined by

$$
c_{k}(t)=[2 E(t) a(t)-b(t)]\left\langle\left\langle\lambda^{k}\right\rangle\right\rangle=\rho^{2} A(t)\left\langle\left\langle\lambda^{k}\right\rangle\right\rangle .
$$

Here $A(t)$ is the acceptance rate defined in Eq. (46). Note that the rate variation of the moment $h_{k}$ depends linearly on the moments $h_{k}$ and $h_{k+1}$, but the coefficients $a(t), b(t)$, and $c(x, t)$ are nonlinear functions of $h_{1}=-\frac{1}{2} E$ and 
$B_{1}=h_{2}-h_{1}^{2}$ (second cumulant). It is thus reasonable to expect that the Monte Carlo dynamics is determined by the evolution of the first two moments. By means of the moments generating function $g(x, t)$ of Eq. (10), we obtain from Eq. (51)

$$
\frac{\partial g(x, t)}{\partial t}=a(t) \frac{\partial g}{\partial x}+b(t) g+c(x, t),
$$

where the time-dependent quantities $a, b$ are functions of the two first moments $E(t)$ and $h_{2}(t)$ (whose relation to $g$ is indicated below) defined in (43) and

$$
c(x, t)=[2 E(t) a(t)-b(t)]\left\langle\left\langle e^{x \lambda}\right\rangle\right\rangle=\rho^{2} A(t)\left\langle\left\langle e^{x \lambda}\right\rangle\right\rangle .
$$

As in the case of Langevin dynamics, $g(x, t)$ is a solution of Eq. (54) plus the following initial, boundary, and subsidiary conditions:

$$
\begin{gathered}
g(x, 0)=g_{0}(x), \\
g(0, t)=1, \\
\left.\frac{\partial g}{\partial x}\right|_{x=0}=-2 E(t), \\
\left.\frac{\partial^{2} g}{\partial x^{2}}\right|_{x=0}=h_{2}(t) .
\end{gathered}
$$

The second condition is the spherical one and the third and fourth define the first and second moments of the set $h_{k}$. This linear partial differential equation can be readily solved with the method of characteristics. The general solution for a partial differential equation of the previous type, Eq. (54), is shown in Appendix B.

\section{Correlation and response functions}

In order to find the dynamical equation for the set of correlation functions, Eq. (20), we perform a similar computation as has been done for the moments $h_{k}$.

The elementary move, Eq. (30), at time $t$ induces a change $\Delta E^{*}$ and $\Delta C_{k}^{*}$ in the energy and the $k$ moment of the correlation function $C_{k}\left(t^{\prime}, t\right)$ (in what follows we take $t^{\prime}<t$ ) defined in Eq. (20),

$$
\begin{gathered}
\Delta E^{*}=-\frac{1}{\sqrt{N}} \sum_{\lambda} \lambda \sigma_{\lambda}(t) r_{\lambda}(t)-\frac{1}{2 N} \sum_{\lambda} \lambda r_{\lambda}(t)^{2}, \\
\Delta C_{k}^{*}=\frac{1}{\sqrt{N}} \sum_{\lambda} \lambda^{k} \sigma_{\lambda}\left(t^{\prime}\right) r_{\lambda}(t) .
\end{gathered}
$$

The probability of having a change of the energy $\Delta E$ and the correlation $\Delta C_{k}$ is given by the joint probability

$$
P\left(\Delta E, \Delta C_{k}\right)=\int \delta\left(\Delta E-\Delta E^{*}\right) \delta\left(\Delta C_{k}-\Delta C_{k}^{*}\right) \delta\left(\Delta h_{0}\right) \prod_{\lambda}\left[p\left(r_{\lambda}\right) d r_{\lambda}\right]
$$

Using the integral representation of the $\delta$ function, retaining only the terms of order $1 / N$, and performing all the Gaussian integrals we find $P\left(\Delta E, \Delta C_{k}\right)=P(\Delta E) P\left(\Delta E \mid \Delta C_{k}\right)$, where

$$
P\left(\Delta E \mid \Delta C_{k}\right)=\frac{1}{\sqrt{2 \pi \rho^{2}\left(h_{2 k}^{\prime}-C_{k}^{2}-\frac{D_{k}^{2}}{B_{1}}\right)}} \exp \left(-\frac{\left\{\Delta C_{k}+\left(\rho^{2} / 2\right) C_{k}+\left(D_{k} / B_{1}\right)\left[\Delta E-\left(\rho^{2} / 2\right) h_{1}\right]\right\}^{2}}{2 \rho^{2}\left(h_{2 k}^{\prime}-C_{k}^{2}-\left(D_{k}^{2} / B_{1}\right)\right)}\right),
$$

where $h_{2 k}^{\prime}=h_{2 k}\left(t^{\prime}\right), D_{k}\left(t^{\prime}, t\right)=C_{k+1}\left(t^{\prime}, t\right)+2 E(t) C_{k}\left(t^{\prime}, t\right)$, and $P(\Delta E)$ is given by Eq. (38).

To solve the equation of motion for the $C_{k}\left(t^{\prime}, t\right)$ we write its average variation at time $t$ over the accepted changes of configuration,

$$
\frac{\partial C_{k}\left(t^{\prime}, t\right)}{\partial t}=\overline{\Delta C_{k}\left(t^{\prime}, t\right)}=\int_{-\infty}^{\infty} \Delta C_{k} d\left(\Delta C_{k}\right)\left(\int_{-\infty}^{0} d(\Delta E) P\left(\Delta C_{k}, \Delta E\right)+\int_{0}^{\infty} d(\Delta E) \exp (-\beta \Delta E) P\left(\Delta C_{k}, \Delta E\right)\right) .
$$

Using the formulas of Appendix A we get the result

$$
\frac{\partial C_{k}\left(t^{\prime}, t\right)}{\partial t}=\frac{a(t)}{2} C_{k+1}\left(t^{\prime}, t\right)+\frac{b(t)}{2} C_{k}\left(t^{\prime}, t\right),
$$

where $a(t), b(t)$ have been previously defined in Eq. (43).

Equation (59) is solved with the initial condition $C_{k}\left(t^{\prime}, t^{\prime}\right)=h_{k}\left(t^{\prime}\right)$ [once the time evolution of the $h_{k}(t)$ has been obtained solving the preceding hierarchy corresponding to the set of moments $h_{k}$.
In order to close the previous hierarchy of equations we introduce the generating function $K\left(x, t^{\prime}, t\right)$ of Eq. (22) which yields the generalized two-time moments $C_{k}\left(t^{\prime}, t\right)=\left[\partial^{k} K\left(x, t^{\prime}, t\right) / \partial x^{k}\right]_{x=0}$. The $K\left(x, t^{\prime}, t\right)$ satisfies the partial differential equation

$$
\frac{\partial K}{\partial t}=\frac{a(t)}{2} \frac{\partial K}{\partial x}+\frac{b(t)}{2} K
$$


together with $K\left(x, t^{\prime}, t^{\prime}\right)=g\left(x, t^{\prime}\right)$. The solution can be found by the method of characteristics. A similar procedure can be used to obtain the generating function for the moments of the response function, Eq. (24). In the Monte Carlo dynamics the equivalent quantity is given by the moments

$$
G_{k}\left(t^{\prime}, t\right)=\lim _{\Delta \rightarrow 0} \frac{1}{N \Delta} \sum_{\lambda} \lambda^{k} m_{\lambda}\left(t^{\prime}\right) \sigma_{\lambda}(t)
$$

where $\Delta$ measures the intensity of an applied staggered field $m_{\lambda}$. This quantity measures the correlation between the spin configuration at time $t$ and a small staggered magnetic field $m_{\lambda}\left(t^{\prime}\right)$ applied at a previous time $t^{\prime}$. Equation (61) is the analog of the correlation between the spin configuration at time $t$ and the noise at time $t^{\prime}$ in the Langevin approach. The staggered magnetic field $m_{\lambda}$ is an uncorrelated annealed random field taken from a Gaussian distribution of variance $\Delta$,

$$
P\left(m_{\lambda}\right)=\left(2 \pi \Delta^{2}\right)^{-1 / 2} \exp \left(-\frac{m_{\lambda}^{2}}{2 \Delta^{2}}\right)
$$

at each elementary move in the MC dynamics. The calculation of the response function proceeds in the following way: We compute the probability distribution for the variation of the $G_{k}\left(t^{\prime}, t\right)$ when a small staggered magnetic field $m_{\lambda}\left(t^{\prime}\right)$ is applied at an earlier time $t^{\prime}$ in an elementary move, Eq. (30), at time $t$. We compute the joint probability distribution of the variation $\Delta G_{k}\left(t^{\prime}, t\right)$ for an elementary move at time $t$,

$$
\begin{aligned}
P\left(\Delta E, \Delta G_{k}\right)= & \int \delta\left(\Delta E-\Delta E^{*}\right) \delta\left(\Delta G_{k}-\Delta G_{k}^{*}\right) \delta\left(\Delta h_{0}\right) \\
& \times \prod_{\lambda}\left[p\left(r_{\lambda}\right) P\left(m_{\lambda}\left(t^{\prime}\right)\right) d r_{\lambda} d m_{\lambda}\left(t^{\prime}\right)\right],
\end{aligned}
$$

where $\Delta h_{0}$ is given in Eq. (34) and the variations of energy and $G_{k}$ are given by

$$
\begin{gathered}
\Delta E^{*}=-\frac{1}{\sqrt{N}} \sum_{\lambda} \lambda \sigma_{\lambda}(t) r_{\lambda}(t)-\frac{1}{2 N} \sum_{\lambda}\left[\lambda r_{\lambda}(t)^{2}\right. \\
\left.-m_{\lambda}\left(t^{\prime}\right) r_{\lambda}(t)\right], \\
\Delta G_{k}^{*}=\frac{1}{\sqrt{N}} \sum_{\lambda} \lambda^{k} m_{\lambda}\left(t^{\prime}\right) r_{\lambda}(t) .
\end{gathered}
$$

Finally, we perform the limit $\Delta$ tending to zero for the intensity of the staggered magnetic field. Performing similar computations as in the correlation function case we obtain for the average variation $\partial G_{k}\left(t^{\prime}, t\right) / \partial t$ (in the region of times $\left.t^{\prime}<t\right)$ Eq. (59), substituting $C_{k}$ for $G_{k}$. Note that the difference between the hierarchy of equations associated with the correlation and the response functions lies in the region of times $t^{\prime}>t$ where $G_{k}\left(t^{\prime}, t\right)=0$ while $C_{k}\left(t^{\prime}, t\right)=C_{k}\left(t, t^{\prime}\right)$.

The partial differential equation associated with the generating function, Eq. (25), is in this case

$$
\frac{\partial \Gamma}{\partial t}=a(t) \frac{\partial \Gamma}{\partial x}+b(t) \Gamma+\langle\langle\exp (x \lambda)\rangle\rangle \delta\left(t-t^{\prime}\right) .
$$

The partial differential equations (60) and (65) can be also readily solved with the method of characteristics (see Appen$\operatorname{dix}$ B) as was done for in case of the one-time quantities. Note that the equations for the generating functions $g, K, \Gamma$ [Eqs. (54), (60), (65)] are formally the same as in the Langevin approach, Eqs. (11), (23), (26), with the time-dependent parameters $a(t), b(t), c(x, t)$ given by

$$
\begin{aligned}
a_{\text {Lang }}(t) & =2, b_{\text {Lang }}(t)=2(2 E-T), \quad c_{\text {Lang }}(x, t) \\
& =2 T\langle\langle\exp (x \lambda)\rangle\rangle .
\end{aligned}
$$

The main difference between the MC dynamics and the Langevin dynamics relies on the simpler time dependence of the coefficients $a, b, c$ in the last case. This makes the largetime behavior of the Langevin dynamics exactly soluble (see Refs. 24,25 ) while this is a very complicated task in the MC case.

\section{ANALYSIS OF THE MONTE CARLO DYNAMICAL EQUATIONS}

In this section we proceed to solve the resulting dynamical equations for the MC dynamics. First we analyze the equilibrium dynamics, showing that it coincides with the Langevin dynamics by an appropriate rescaling of time. Then we study the off-equilibrium behavior contained in the MC dynamics at finite and zero temperature. Since it is also our purpose to test the correctness of the solution of our equations, we will compare the theory with real Monte Carlo numerical simulations. Moreover, we will compare the resulting dynamics with that expected in the Langevin case.

\section{A. Equilibrium Monte Carlo dynamics}

Dynamical equations for one-time and two-time quantities can be readily solved at equilibrium. Now the observables $h_{k}$ are independent of time and the two-time functions $C_{k}\left(t^{\prime}, t\right), G_{k}\left(t^{\prime}, t\right)$ only depend on the differences of time $t-t^{\prime}$. It is easy to check that the coefficients $a(t), b(t), c(x, t)$ of Eqs. (43),(55) are time independent and given by

$$
\begin{gathered}
a^{\mathrm{eq}}=\rho^{2} \beta \operatorname{Erf}\left(\alpha^{\mathrm{eq}}\right), \\
b^{\mathrm{eq}}=\rho^{2} \beta(2 E-T) \operatorname{Erf}\left(\alpha^{\mathrm{eq}}\right), \\
c^{\mathrm{eq}}(x)=\rho^{2}\langle\langle\exp (x \lambda)\rangle\rangle \operatorname{Erf}\left(\alpha^{\mathrm{eq}}\right),
\end{gathered}
$$

with

$$
\alpha^{\mathrm{eq}}=-\frac{\rho E^{\mathrm{eq}}}{\sqrt{2 B_{1}^{\mathrm{eq}}}}=\rho \sqrt{\frac{(2 \beta-1)}{8}} .
$$

These coefficients are the same as for the Langevin dynamics except for a rescaling of time, $t \rightarrow t^{\prime}=\left[\rho^{2} \beta \operatorname{Erf}\left(\alpha^{\mathrm{eq}}\right) / 2\right] t$. Also one can show [using Eq. (46)] that the acceptance rate is given by

$$
A^{\mathrm{eq}}=\operatorname{Erf}\left(\alpha^{\mathrm{eq}}\right) .
$$

Using the previous rescaling of time the equilibrium MC dynamical equations coincide with the Langevin ones. ${ }^{26}$ 


\section{B. Monte Carlo simulations}

In order to check our analytical results for the MC dynamics we have performed some MC simulations for finite sizes. To simulate enough large sizes we worked in the basis of eigenvectors $\sigma_{\lambda}$. In this way all the information about the quenched disorder is fully contained in the spectrum of eigenvalues $\lambda$, which occupies much less memory than the full interaction Gaussian matrix $J_{i j}$. The set eigenvalues $\lambda$ is chosen according to the semicircular law, Eq. (6). Typically we start from a random initial configuration $\sigma_{\lambda}= \pm 1$ which fulfills the spherical constraint. Then we perform a small rotation of this configuration, $\left\{\sigma_{\lambda}\right\} \rightarrow\left\{\sigma_{\lambda}+r_{\lambda} / \sqrt{N}\right\}$ [Eq. (30)] where the $r_{\lambda}$ are random numbers extracted from the Gaussian distribution, Eq. (31). To keep invariant the spherical constraint we normalize the length of the vector $\left\{\sigma_{\lambda}\right\}$ in order to make it of length 1 . The resulting change of energy, Eq. (32), is computed and accepted with probability $\operatorname{Min}(1, \exp (-\beta \Delta E))$. We repeat this process for the new configuration and so on. A MC step corresponds to $N$ rotations. Because an elementary move involves a global change of the configuration $\left\{\sigma_{\lambda}\right\}$, this algorithm is $N$ times slower than a usual MC algorithm with only local changes. We are able to simulate relatively large sizes in the range $N=500-2000$ in a reasonable amount of computer time.

\section{Finite-temperature dynamics}

In case of finite temperature it is relatively easy to solve MC equations in the large-time limit. All the information on the dynamics is contained in the time evolution of the coefficients $a(t), b(t), c(x, t)$ which monotonically converge to their equilibrium values. Then we expect that the dynamics converges to the Langevin dynamics in the large-time limit except by a rescaling of the time

$$
t \rightarrow t^{\prime}=\frac{\rho^{2} \beta \operatorname{Erf}\left(\alpha^{\mathrm{eq}}\right)}{2} t .
$$

At finite temperature and according to the magnitude of the parameter $\rho$ we distinguish two different regimes depending if the acceptance rate is large or small. These two limits differ in the magnitude of the time scale above which Langevin behavior is recovered.

\section{Case $\rho<1$}

In this case $\operatorname{Erf}(\alpha) \sim O(1)$ since $\alpha$ is small; i.e., the acceptance is always large.

Note that in the particular limit $\rho$ tending to zero the Langevin dynamics is recovered with a rescaling of time

$$
t \rightarrow t^{\prime}=\frac{\rho^{2} \beta}{2} t .
$$

In Fig. 1 we show the decay of the energy for different temperatures for a given value of $\rho=0.1$ by numerically solving Eq. (B3). The theoretical prediction is compared with real Monte Carlo simulation results for $N=500$ spins. In the figure we also show the asymptotic large-time behavior in the Langevin case, $E(t)-E^{\mathrm{eq}} \sim \frac{3}{8} t^{-1}$ (see Ref. 25) with the rescaling of time, Eq. (70).

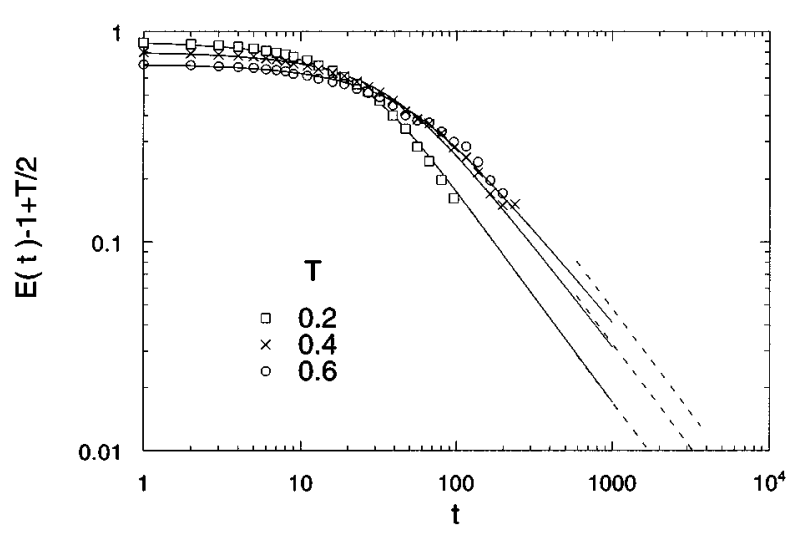

FIG. 1. Relaxation of the energy $E(t)-E^{\mathrm{eq}}$ as a function of time for $\rho=0.1$ and temperatures $T=0.2,0.4,0.6$. The points are the MC simulations data $(N=500$, one sample), the lines are the analytical solution of the MC dynamical equations, and the dashed line is the large-time behavior described by the Langevin dynamics rescaling the time as described in the text.

Results for the correlation function $C_{0}\left(t_{w}, t_{w}+t\right)$ $=K\left(0, t_{w}, t_{w}+t\right)$ are shown in Fig. 2 for different values of $t_{w}=1,10,100,1000$ at the temperature $T=0.4$ with $\rho=0.1$ obtained by numerically solving Eq. (B4). Again we compare the solution with real MC simulations for $N=500$ spins. In the figure we also show the asymptotic large-time behavior in the Langevin case, $C\left(t_{w}, t_{w}+t\right) \sim t^{-3 / 4}$ (see Ref. 25).

Note that in the dynamical regime shown in Fig. 2 there is no evidence of a plateau or $\beta$-relaxation process in the correlation function. This process is a signature of the existence of a short-time regime (fluctuation dissipation theorem) where the system is locally in equilibrium and the fluctuation-dissipation theorem applies [i.e., the $\left.C_{0}\left(t_{w}, t_{w}+t\right) \simeq C_{0}(t)\right]$. The reason why the $\beta$-relaxation process is not seen in Fig. 2 is that the values of $t_{w}$ shown there are too small. In the Langevin dynamics this regime usually appears for large values of $t_{w}$ which become much larger in the MC case since we have to rescale the time by the parameter (70) (the constant we have to rescale the time in Fig. 2 is approximately 87; i.e., $87 \mathrm{MC}$ steps corre-

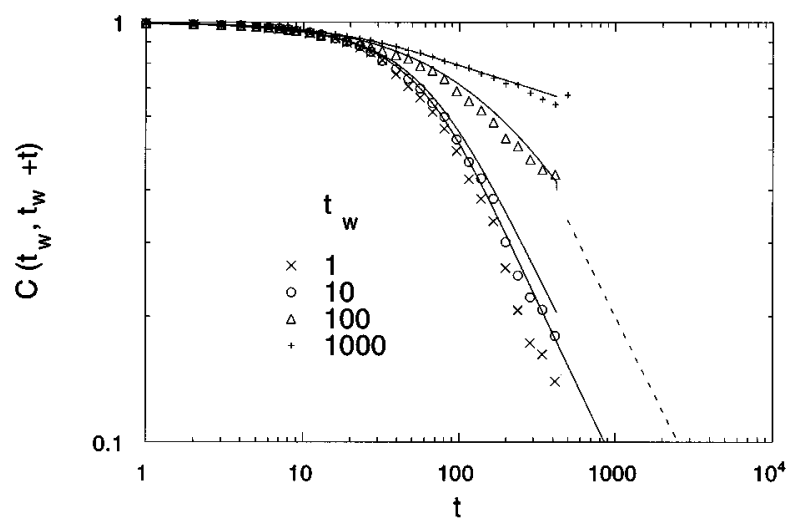

FIG. 2. $C_{0}\left(t_{w}, t_{w}+t\right)$ with $\rho=0.1, \quad T=0.4$ for $t_{w}=1,10,100,1000$. The points are the MC simulations data ( $N=500$, one sample), the lines are the analytical solution of the MC dynamical equations, and the dashed line is the large-time behavior described by the Langevin dynamics rescaling the time as described in the text. 


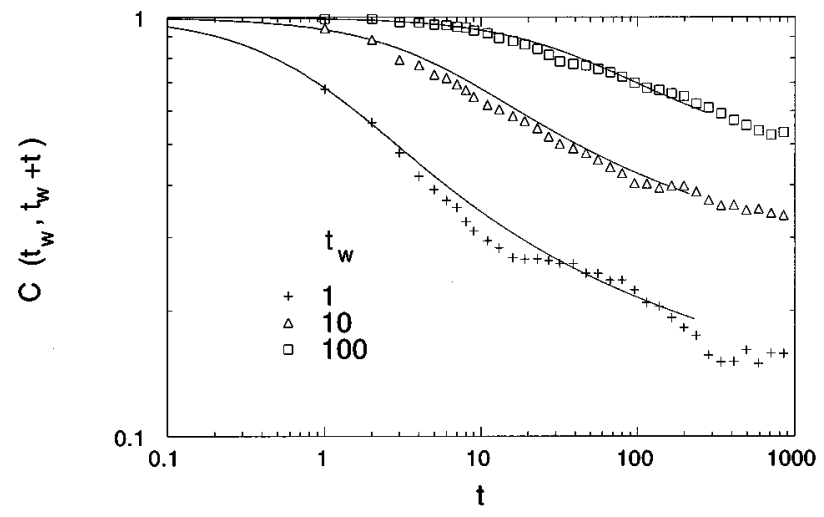

FIG. 3. $C_{0}\left(t_{w}, t_{w}+t\right)$ with $\rho=5, T=0.4$ for $t_{w}=1,10,100$. The points are the MC simulations data $(N=500$, data averaged over 5 samples) and the lines are the analytical solution of the MC dynamical equations.

spond to one unit of time in the Langevin dynamics). In this regime $\rho \ll 1$, because $\operatorname{Erf}(\alpha)$ is finite, we expect the $t_{w}$ necessary to observe the plateau to scale like $\rho^{-2}$ times the needed time in the Langevin case which requires quite large values of $t_{w}$ in the MC case. This off-equilibrium regime observed in the case $\rho \ll 1$ where the acceptance rate is large is very similar to that observed in models with a continuous breaking of the replica symmetry, like the SK model ${ }^{3}$ or finite-dimensional spin glasses ${ }^{2,22}$ where no evidence of a plateau is found in the region of values of $t_{w}$ explored.

\section{Case $\rho>1$}

In this case $\operatorname{Erf}(\alpha) \sim e^{-\alpha^{2}} / \alpha$ since $\alpha$ is large; i.e., the acceptance is always very small. In this regime the rescaled time constant, Eq. (70), is again small because $\operatorname{Erf}(\alpha)$ is small (it decreases with $\rho$ like $\rho e^{-\rho^{2}}$ ). For values of $t_{w} \ll \exp \left(\rho^{2}\right) / \rho$ the system is in the short-time regime where a plateau is present and aging is present [i.e., the $C_{0}\left(t_{w}, t_{w}+t\right)$ strongly depends on $\left.t_{w}\right]$. This is clearly appreciated in Fig. 3 where we show the $C_{0}\left(t_{w}, t_{w}+t\right)$ for different values of $t_{w}=1,10,100,1000$ at $T=0.4, \rho=5$, and the numerical solution of Eq. (B4). In the regime $t_{w} \ll \exp \left(\rho^{2}\right) / \rho$ there is a plateau which increases with $t_{w}$ and eventually converges to the value $q_{\mathrm{EA}}=1-T$ for times $t_{w}$ of order $\exp \left(\rho^{2}\right) / \rho$. This is a transient regime where the decay to the plateau does not satisfy time-translational invariance. No equivalent regime is found in the Langevin dynamics of the spherical Sherrington-Kirkpatrick model.

The behavior shown in Fig. 3 is very similar to that found in systems with strong freezing in the low-temperature regime where the acceptance rate is quite low. Generally these are systems with one step of replica symmetry breaking like the spherical or Ising $p$-spin model $(p>2), 9,29$ the Potts glass model with $p$ states $(p>4),{ }^{30}$ the random orthogonal model (ROM) ${ }^{31}$ and frustrated systems without quenched disorder like the Bernasconi model ${ }^{32}$ and other types of models. ${ }^{33}$ All these systems are characterized by the presence of a dynamics with very low acceptance rate and the existence of a dynamical transition different from the statical one. While the statics of the aforementioned class of models is much different from that of the spherical SK model the dynamics shows interesting similarities.

\section{Zero-temperature dynamics}

In this subsection we are interested in extracting the largetime behavior of the dynamical equations at zero temperature. There is no reason in the zero-temperature case to get the same large-time behavior as in the Langevin case, the time-rescaling equation (70) being ill defined. Because in the zero-temperature regime the acceptance rate goes monotonically to zero (i.e., the parameter $\alpha$ diverges), the coefficients $a(t), b(t), c(x, t)$ in Eqs. (43),(55) have a nontrivial largetime limit. Then we expect a dynamical regime much different from that predicted in the Langevin dynamics. In fact, we will see that there is no limiting case in which the Langevin dynamics is found.

At $T=0$ the coefficients $a(t), b(t), c(x, t)$ of Eqs. (43),(55) become

$$
\begin{gathered}
a_{T=0}(t)=-\frac{2 \alpha e^{-\alpha^{2}}}{E \sqrt{\pi}}, \\
b_{T=0}(t)=-\left(\frac{\rho^{2} \operatorname{Erf}(\alpha)}{2}+\frac{4 \alpha e^{-\alpha^{2}}}{\sqrt{\pi}}\right), \\
c_{T=0}(x, t)=\frac{1}{2} \rho^{2}\left\langle\left\langle e^{x \lambda}\right\rangle\right\rangle \operatorname{Erf}(\alpha) .
\end{gathered}
$$

Also Eq. (45) for the acceptance rate reads

$$
A(t)=\frac{\operatorname{Erf}(\alpha)}{2} .
$$

Note that the large-time dynamics is governed by the time-dependent parameter $\alpha$ which diverges in the infinitetime limit. Unfortunately the dependence of the coefficients on $\alpha$ is strongly nonlinear, which makes the mathematical treatment of the dynamical equations highly nontrivial. However, we can derive a nonlinear equation for the energy alone by means of the adiabatic approximation explained in the next subsection. Although a rigorous derivation of the adiabatic approximation is not known to us, the resulting equation yields an excellent approximation to the large-time behavior as given by the numerical solutions of the exact dynamical equations.

We can analyze qualitatively how the system evolves at zero temperature. Suppose the system starts from a random initial configuration $\sigma_{i}= \pm 1$ such that $E(t=0)=0$ and $B_{1}(t=0)=1$. The energy monotonically decreases to the ground-state energy $E=-J_{\max } / 2=-1$ while $B_{1}$ decreases also to zero. In the large-time limit $\alpha$ diverges and the acceptance rate goes to zero. There are two different regimes in the dynamics. The first one is an initial regime where $\alpha$ is small and the acceptance rate is nearly $1 / 2$. This corresponds to a Gaussian $P(\Delta E)$ [Eq. (38)] with width $\rho \sqrt{B_{1}}$ larger than the position of its center $\left(\rho^{2} E\right)$. In this case, the changes of the configuration which increase or decrease the energy have the same probability. The energy decreases fast in this regime because the acceptance is large. The second regime appears when $B_{1}$ is so small in order that $\alpha$ becomes large. 


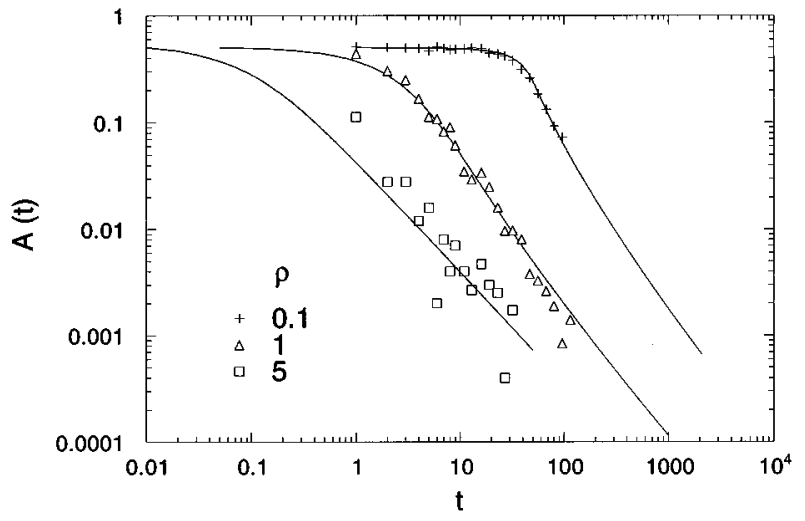

FIG. 4. Acceptance rate $A(t)$ at $T=0$ as a function of time for $\rho=0.1,1,5$ obtained by solving the MC dynamical equations (lines) compared with MC simulations (points) $(N=500$, one sample).

In this case the acceptance is very small [it goes like $\left.\exp \left(-\alpha^{2}\right) / \alpha\right]$ and the dynamics is strongly slowed down. The system goes very slowly to equilibrium. It is in this second regime where the adiabatic approximation developed in the next subsection applies.

The adiabatic approximation shows that the large-time behavior in the Monte Carlo case is quite different from the Langevin case. This is not a surprise. Even for the simple harmonic oscillator, one can show that at zero temperature the dynamics is different in the MC and in the Langevin cases. ${ }^{34}$ This is related to the nonergodic nature of the dynamics at zero temperature in which only the changes which decrease the energy are accepted. This is clearly shown in Figs. 4, 5, 6, and 7. In Figs. 4 and 5 we show the acceptance rate and energy as a function of time for three different values of $\rho$ by numerically solving the off-equilibrium equation (B3) with the coefficients (72).

We note two important results. First, as previously said, we observe that $\alpha$ is the parameter which governs the dynamics. More concretely Fig. 4 shows that the magnitude of $\alpha$ separates two different regimes. In the regime $\alpha \ll 1$ the acceptance rate is 0.5 while it falls down rapidly to zero in the regime $\alpha \gg 1$. Second, the large-time behavior of the energy shown in Fig. 5 is strikingly different from that expected in the Langevin dynamics at zero temperature where

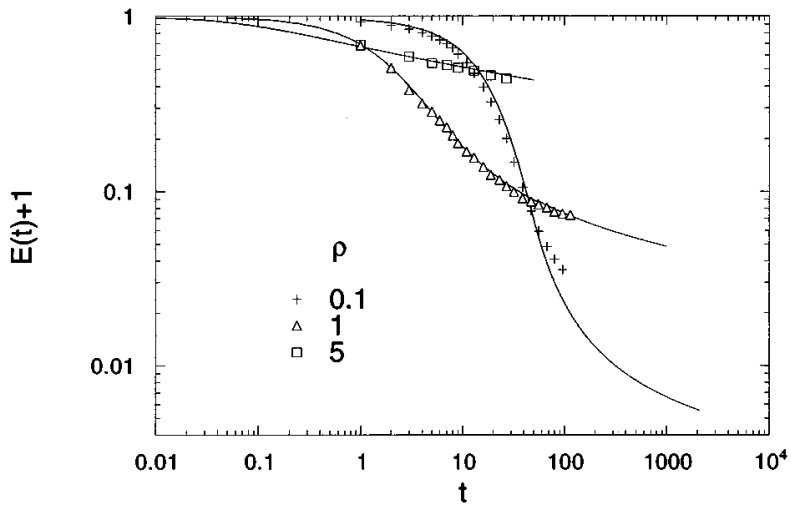

FIG. 5. Energy $E(t)+1$ at $T=0$ as a function of time for $\rho=0.1,1,5$ obtained solving the MC dynamical equations (lines) compared with MC simulations (points) $(N=500$, one sample).

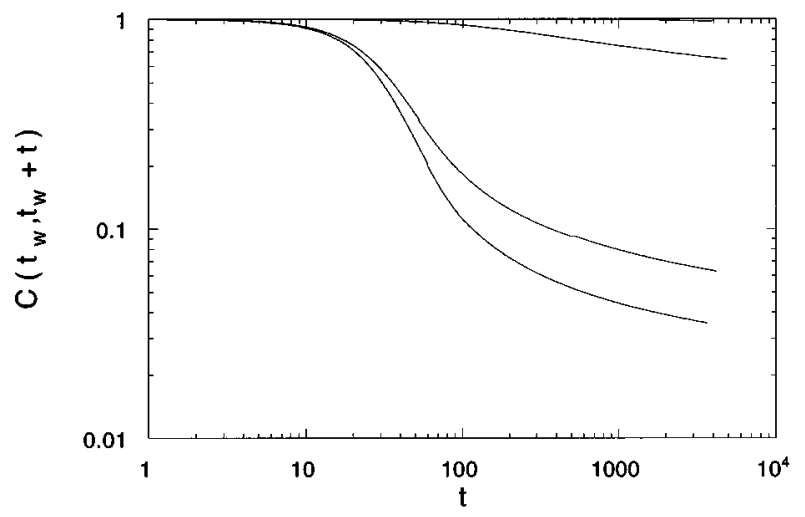

FIG. 6. $C_{0}\left(t_{w}, t_{w}+t\right)$ at $T=0$ with $\rho=0.1$ for $t_{w}=1,10,100,1000$ (from bottom to top) obtained solving the MC dynamical equations.

$E(t)+1 \simeq O(1 / t) .{ }^{25}$ The energy decays like $1 / \ln (t)$ (plus corrections) as shown below.

Figure 6 shows the $C_{0}\left(t_{w}, t_{w}+t\right)$ as a function of $t$ for four different values of $t_{w}$ by numerically solving the offequilibrium equation (B4) with the coefficients (72). Note that the large- $t$ behavior of the $C\left(t_{w}, t_{w}+t\right)$ is strikingly different in the $\mathrm{MC}$ and the Langevin cases since no trace of the power law decay $t^{-3 / 4}$ is observed and the $t / t_{w}$ scaly behavior is lost. The correlation function strongly freezes (i.e., remains very close to 1 ) for values of $t_{w}$ such that the acceptance rate at that time has already jumped to zero (see Fig. 4). This is reasonable because a very low number of accepted changes implies a very small change of the correlation function.

\section{E. Adiabatic approximation}

We now discuss the adiabatic approximation which gives the correct asymptotic large-time behavior of the energy and the acceptance rate at zero temperature.

The equation of the energy, Eq. (42), can be written as a function only of the parameter $\alpha$,

$$
\frac{1}{E} \frac{\partial E}{\partial t}=-\frac{1}{2} \rho^{2} \operatorname{Erf}(\alpha)+\frac{\rho^{2} e^{-\alpha^{2}}}{2 \alpha \sqrt{\pi}} .
$$

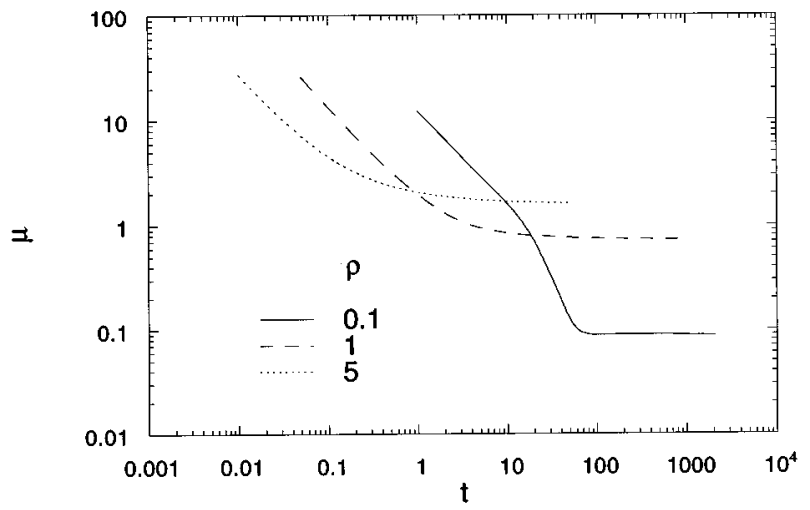

FIG. 7. Ratio $-B_{1} /[2 E(E+1)]$ as a function of time at $T=0$ for different values of $\rho$. The large-time limit yields the effective parameter $\mu^{*}$. 
This is an exact equation for the energy. Unfortunately we cannot solve it because we do not know the time evolution of $\alpha$. Once $\alpha$ is known we also know the time evolution of the energy, hence also all the moments $h_{k}$. In this sense, the parameter $\alpha$, and consequently the acceptance rate, fully determines the dynamics. In the large-time regime, where $\alpha$ is very large, we can expand the error function $\operatorname{Erf}(\alpha) \simeq\left(e^{-\alpha^{2}} / \sqrt{\pi} \alpha\right)\left(1-1 / 2 \alpha^{2}\right)$ and we get the simple equation

$$
\frac{1}{E} \frac{\partial E}{\partial t}=\frac{\rho^{2} e^{-\alpha^{2}}}{2 \sqrt{\pi} \alpha^{3}} .
$$

We solve this equation using an adiabatic approximation which turns out to be the correct large-time solution as one can check numerically solving Eq. (B3). We replace $\alpha$ by an effective parameter which depends on off-equilibrium quantities via a quasiequilibrium relation. In our case we use the quasiequilibrium relation (68) with $\beta=1 / T=1 / \mu^{*}(E+1)$, where $\mu^{*}$ is a renormalized parameter which has the physical meaning of an effective temperature. Note that $\mu^{*}=2$ in the previous equation yields the equilibrium relation $E=-1+T / 2$ in the low-temperature phase. Substituting in (75) and using $B_{1}=-2 \mu^{*} E(E+1)$ we obtain, in the largetime limit (i.e., $E \simeq-1$ ) and neglecting higher orders in $E+1$,

$$
\frac{\partial E}{\partial t}=-\frac{2}{\rho \sqrt{\pi}}\left[\mu^{*}(E+1)\right]^{3 / 2} \exp \left(-\frac{\rho^{2}}{4 \mu^{*}(E+1)}\right) .
$$

In principle, $\mu^{*}$ is an unknown parameter. In the simplest adiabatic approximation $\mu^{*}=2$. This corresponds to equipartitioning in the surface of constant energy where the equilibrium relation $B_{1}=-4 E(E+1)$ is fulfilled at all times. It is possible to show ${ }^{34}$ that for $\mu^{*}=2 \mathrm{Eq}$. (76) is the equation of the energy for a simple harmonic oscillator with Hamiltonian $\mathcal{H}=\frac{1}{2} m \omega^{2} x^{2}$, where $m \omega^{2}=\lambda^{\max }=2$. Physically this means that the system relaxes as a simple harmonic oscillator with a value of $m \omega^{2}$ determined by the maximum eigenvalue of the spectrum. Physically a value of $\mu^{*}<2$ means that the system relaxes as an effective single harmonic oscillator with effective mass $m \omega^{2}=4 / \mu^{*}$ larger than the single oscillator mass $m \omega^{2}=2 .^{34}$

The large-time solution of Eq. (76) can be easily worked out. One finds that the parameter $\alpha$ diverges like $[\ln (t)]^{1 / 2}$, the energy decays like $E(t)=-1+O(1 / \ln (t))$, and the acceptance rate goes like $A(t) \simeq 1 / t$. All these quantities have nontrivial subdominant corrections. In Fig. 7 we show the effective parameter $\mu^{*}=-B_{1} / 2 E(E+1)$ as a function of time obtained by solving Eq. (B3) for different values of $\rho$. Note that the effective parameter $\mu^{*}$ converges to a timeindependent value in the asymptotic large-time limit. By numerically solving the adiabatic equation (76) we have checked that the relaxation of the energy is in excellent agreement with the numerical solution of Eq. (B3). This is a check of the adiabatic approximation which yields the values $\mu^{*} \simeq 0.087,0.72,1.65$ for $\rho=0.1,1,5$, respectively. Note that these quoted values of $\mu^{*}$ are smaller than 2 .

\section{CONCLUSIONS}

In this work we have presented the analytical solution of the Monte Carlo dynamics of the spherical SherringtonKirkpatrick model. This is a very simple spin-glass model where full computations can be carried out. In order to analytically solve the MC dynamics we have used the technique of the generating function. The generating function allows us to close the dynamical equations in a similar way to how it has been done in other glassy models without quenched disorder. The present work is a step towards the use of this powerful technique in the spin-glass context. As an example, we have solved the Langevin dynamics of the spherical SK model. In the context of the MC dynamics, the generating function has been used to derive closed expressions for onetime quantities, like the generalized moments $h_{k}$, and for two-time quantities such as the correlation or response function. The formalism of the generating function may be used to compare the results obtained with both dynamics, $\mathrm{MC}$ and Langevin.

By solving such a simple model, we lose the subtleties of having a replica-broken low-temperature phase where the presence of a large number of metastable states makes dynamics much more interesting. ${ }^{35}$ Nevertheless, our results for the MC dynamics make it clear how relevant for offequilibrium dynamics is the acceptance rate, a quantity which is absent in Glauber or Langevin dynamics. Since a major part of the numerical work on glassy systems employs MC dynamics, its analytical study is of the utmost importance to be used as a guide when trying to extract conclusions from numerics.

One of the most important results which emerges from this work concerns the importance of the acceptance rate in the off-equilibrium dynamics. We have seen that the main differences between the Langevin and the MC dynamics appear whenever this rate is small and for times not too large. The Langevin dynamics is obtained in the limit $\rho \rightarrow 0$ with an effective rescaling of time $t \rightarrow t^{\prime}=t \rho^{2} \beta / 2$ and the acceptance ratio is always 1 [cf. Eqs. (43) and (55)]. For finite $\rho$ and finite temperatures we have seen that the Langevin regime is found for times $t \simeq \rho^{-2}$ when $\rho$ is small and for times $t \simeq \exp \left(\rho^{2}\right) / \rho$ when $\rho$ is large. In the intermediate regimes very many different behaviors are observed, especially for $\rho>1$ where the acceptance is small. The dynamics observed in Fig. 3 reminds one a lot of what is observed in models with one step of breaking (see, for instance, Refs. 36,37). Furthermore, at zero temperature, the MC dynamics yields a completely new behavior where the system rapidly freezes as soon as the acceptance goes to zero. No analogous regime is found in the case of Langevin dynamics where the transition from finite to zero temperature is smooth.

We would like to comment on three different directions which we think would be interesting to explore. The first concerns the adiabatic approximation which is used to obtain closed equations for one-time moments. As has been shown, this approximation suggests that the system behaves as a system of uncoupled harmonic oscillators with a renormalized mass larger than the maximum mass corresponding to the largest eigenvalue of the Wigner spectrum. Although this suggestive approximation works extremely well, it would be interesting to understand it better. Future research should 
deal with a systematic derivation of the adiabatic approximation for one-time quantities (for instance, understanding the dependence of the effective parameter $\mu^{*}$ as a function of $\rho$ ), and its extension to the study of the long-time behavior of correlation functions. We think that the adiabatic approximation is a consequence of the relevance of entropic effects for the dynamics of the system. In the low-acceptance regime, the system spends a long time searching configurations of lower energy in a very inefficient way. In this regime (not present when the dynamics is Langevin) the adiabatic approximation works very well. More generally one would like to improve it in order to find a systematic way to close the dynamical equations without having to use the full generating function. The work done by Coolen et al. ${ }^{18}$ and that on the backgammon model ${ }^{38}$ is a step in this direction. The second direction to explore is the study of models with one step of replica symmetry breaking (like the $p$-spin spherical spin-glass model $^{9}$ ) using the technique of the generating function. This is an interesting problem whose solution would allow us to obtain closed equations for one-time quantities like the energy and probably a set of higher moments. At present it is not clear whether this is possible or whether we can only obtain equations which relate the correlation and the response functions. ${ }^{9,10}$ Finally we want to mention that the type of differential equations studied here [i.e., semilinear evolution equations like Eq. (54) whose coefficients depend self-consistently on the first moments of the solution] appear often in the study of the dynamics of quite different models in statistical physics. Let us cite among others the problems of synchronization of populations of coupled oscillators, ${ }^{39}$ arrays of Josephson junctions,${ }^{40}$ and plasmas or self-gravitating systems. ${ }^{41,42}$ Often these problems can be described by nonlinear causal equations where the time evolution of an observable at time $t$ depends on all its previous history plus self-consistent relations for functions appearing in the equations. A general mathematical study of these types of equations would be welcome.

\section{ACKNOWLEDGMENTS}

The work of L.L.B., F.G.P., and F.R. has been supported by the DGICYT of Spain under Grant No. PB92-0248 and EC Human Capital and Mobility Programme Contract No. ERBCHRXCT930413. We are grateful to Silvio Franz for a careful reading of the manuscript, and to Conrado PerezVicente and Alex Arenas for stimulating discussions on this and related subjects.

\section{APPENDIX A: THE EQUATION OF MOTION}

In this section we write the general equation of motion associated with the general joint probability distribution

$$
P(\Delta E, \Delta O)=K \exp \left(-\frac{(\Delta E-a)^{2}}{2 b^{2}}\right) \exp \left(-\frac{(\Delta O-c)^{2}}{2 d^{2}}\right),
$$

where $c=e+f(\Delta E-a), a, b, c, d, e, f$ are in general timedependent parameters, $\Delta E$ stands for the energy change, and $\Delta O$ is the change of any observable (generalized moment, correlation, or response function). In the $\mathrm{MC}$ dynamics $a=-\rho^{2} E, b^{2}=\rho^{2} B_{1}$ [with $B_{1}$ given in Eq. (39)], and $c, d, e, f$ depending on the particular observable. The constant $K=\left(4 \pi b^{2} d^{2}\right)^{-1 / 2}$ normalizes the probability distribution.

The equation of motion for the observable $O$ is

$$
\frac{\partial O}{\partial t}=\int_{-\infty}^{\infty} d x \int_{-\infty}^{\infty} d y y P(x, y) w(x),
$$

where $w(x)=\operatorname{Min}\left(1, e^{-\beta x}\right)$ is the Boltzmann factor. Straightforward computations yield

$$
\begin{aligned}
\frac{\partial O}{\partial t}= & \frac{1}{2}\left[e \operatorname{Erf}(\alpha)+\left(e-f \beta b^{2}\right) \exp (-a \beta\right. \\
& \left.\left.+\frac{b^{2} \beta^{2}}{2}\right) \operatorname{Erf}\left(\sqrt{\frac{b^{2} \beta^{2}}{2}}-\alpha\right)\right],
\end{aligned}
$$

where $\alpha=-a /\left(2 b^{2}\right)^{1 / 2}$ and coincides with Eq. (44) with the previously quoted values of $a, b$.

At zero temperature the equation of motion is

$$
\frac{\partial O}{\partial t}=\frac{1}{2}\left(e \operatorname{Erf}(\alpha)-f \sqrt{\frac{2 b^{2}}{\pi}} \exp \left(-\alpha^{2}\right)\right),
$$

with the same previous definition of the parameter $\alpha$.

\section{APPENDIX B: SOLUTION OF THE DIFFERENTIAL EQUATION}

In this section we present the general solution to the generating function $g(x, t)$ in Eq. (54) using the method of characteristics,

$$
\frac{\partial g(x, t)}{\partial t}=a(t) \frac{\partial g}{\partial x}+b(t) g+c(x, t)
$$

In order to solve this equation we make the change of variables $x \rightarrow u(t)=x+\int_{0}^{t} a\left(t^{\prime}\right) d t^{\prime}$ and Eq. (B1) becomes

$$
\frac{d \hat{g}(u, t)}{d t}=b(t) \hat{g}(u, t)+c(u, t),
$$

which is a linear differential equation easily solvable. The final result is

$$
\begin{aligned}
g(x, t)= & g_{0}\left(x+\int_{0}^{t} a\left(t^{\prime}\right) d t^{\prime}\right) B(t) \\
& +B(t) \int_{0}^{t} d t^{\prime} \frac{c\left(x+\int_{t^{\prime}}^{t} a\left(t^{\prime \prime}\right) d t^{\prime \prime}, t^{\prime}\right)}{B\left(t^{\prime}\right)}
\end{aligned}
$$

where $B(t)=\exp \left[\int_{0}^{t} b\left(t^{\prime}\right) d t^{\prime}\right]$ and $g_{0}(x)=g(x, t=0)$ is the initial condition. Once the $g(x, t)$ has been obtained one can also get the generating function of the two-time quantities. For instance, the $K\left(x, t^{\prime}, t\right)$ associated with the correlation function in Eq. (60) is given by

$$
K\left(x, t^{\prime}, t\right)=g\left(x+\frac{1}{2} \int_{t^{\prime}}^{t} a\left(t^{\prime \prime}\right) d t^{\prime \prime}, t^{\prime}\right)\left(\frac{B(t)}{B\left(t^{\prime}\right)}\right)^{1 / 2} .
$$


*Electronic address: bonilla@ing.uc3m.es

†Electronic address: padilla@dulcinea.uc3m.es

*Electronic address: parisi@vaxrom.roma1.infn.it

${ }^{\S}$ Electronic address: ritort@dulcinea.uc3m.es

${ }^{1}$ J. O. Anderson, J. Mattsson, and P. Svedlindh, Phys. Rev. B 46, 8297 (1992).

${ }^{2}$ H. Rieger, J. Phys. A 26, L615 (1993).

${ }^{3}$ L. F. Cugliandolo, J. Kurchan, and F. Ritort, Phys. Rev. B 49, 6331 (1994).

${ }^{4}$ J. Kisker, L. Santen, M. Schreckenberg, and H. Rieger, Phys. Rev. B 53, 6418 (1996).

${ }^{5}$ L. Lundgren, P. Svedlindh, P. Nordblad, and O. Beckman, Phys. Rev. Lett. 51, 911 (1983); E. Vincent, J. Hammann, and M. Ocio, in Recent Progress in Random Magnets, edited by D. H. Ryan (World Scientific, Singapore, 1992), and references therein.

${ }^{6}$ L. C. E. Struik, Physical Aging in Amorphous Polymers and Other Materials (Elsevier, Houston, 1978).

${ }^{7}$ J. P. Bouchaud, J. Phys. (France) I 2, 1705 (1992); J. P. Bouchaud and D. Dean, ibid. 5, 265 (1995).

${ }^{8}$ T. R. Kirkpatrick and D. Thirumalai, Phys. Rev. B 36, 5388 (1987)

${ }^{9}$ A. Crisanti, H. Horner, and H.-J. Sommers, Z. Phys. B 92, 257 (1993).

${ }^{10}$ L. F. Cugliandolo and J. Kurchan, Phys. Rev. Lett. 71, 173 (1993).

${ }^{11}$ S. Franz and M. Mézard, Physica A 210, 48 (1994).

${ }^{12}$ L. F. Cugliandolo and P. Le Doussal, Phys. Rev. E 53, 1525 (1996).

${ }^{13} \mathrm{H}$. Horner (unpublished)

${ }^{14}$ J. P. Bouchaud, L. F. Cugliandolo, J. Kurchan, and M. Mézard (unpublished).

${ }^{15}$ E. Lentheusser, Phys. Rev. A 29, 2765 (1984); T. R. Kirkpatrick, ibid. 31, 939 (1985); W. Gotze and L. Sjogren, Rep. Prog. Phys. 55, 241 (1992).

${ }^{16}$ This connection was realized some time ago by T. R. Kirkpatrick and D. Thirumalai, J. Phys. A 22, L149 (1989).

${ }^{17}$ S. Franz, E. Marinari, and G. Parisi (unpublished).

${ }^{18}$ A. C. C. Coolen and D. Sherrington, J. Phys. A 27, 7687 (1994); A. C. C. Coolen and S. Franz, ibid. 27, 6947 (1994); A. C. C. Coolen, S. Laughton, and D. Sherrington, Phys. Rev. B 53, 8184 (1996).

${ }^{19}$ H. Nishimori and M. Yamana (unpublished).

${ }^{20}$ This equipartitioning approximation has to be carefully introduced in order to obtain good results in the Langevin dynamics of the Gaussian model, as has been shown in G. Parisi (unpublished).

${ }^{21}$ J. M. Kosterlitz, D. J. Thouless, and R. C. Jones, Phys. Rev. Lett. 36, 1217 (1976).

${ }^{22}$ H. Rieger, in Annual Reviews of Computational Physics II, p. 295 (1995); H. Rieger (unpublished).

${ }^{23}$ L. L. Bonilla, F. G. Padilla, G. Parisi, and F. Ritort, Europhys. Lett. 34, 159 (1996).

${ }^{24}$ S. Ciuchi and F. De Pasquale, Nucl. Phys. B300, 31 (1988).

${ }^{25}$ L. F. Cugliandolo and D. S. Dean, J. Phys. A 28, 4213 (1995).

${ }^{26}$ Note that the critical MC dynamics (i.e., the equilibrium dynamics at the critical point $\beta=1$ ) yields the same dynamical exponents as in the Langevin case.

${ }^{27}$ M. L. Mehta, Random Matrices and the Statistical Theory of Energy Levels (Academic, New York, 1967).

${ }^{28}$ M. Mézard, G. Parisi, and M. A. Virasoro, Spin Glass Theory and Beyond (World Scientific, Singapore, 1987); K. H. Fischer and J. A. Hertz, Spin Glasses (Cambridge University Press, Cambridge, England, 1991).

${ }^{29}$ E. Gardner, Nucl. Phys. B257, 747 (1985).

${ }^{30}$ D. J. Gross, I. Kanter, and H. Sompolinsky, Phys. Rev. Lett. 55, 304 (1985).

${ }^{31}$ E. Marinari, G. Parisi, and F. Ritort, J. Phys. A 27, 7647 (1994).

${ }^{32}$ J. Bernasconi, J. Phys. (Paris) 48, 559 (1993); J. P. Bouchaud and M. Mézard, J. Phys. (France) I 4, 1109 (1994); E. Marinari, G. Parisi, and F. Ritort, J. Phys. A 27, 7615 (1994).

${ }^{33}$ E. Marinari, G. Parisi, and F. Ritort, J. Phys. A 28, 327 (1995); L. F. Cugliandolo, J. Kurchan, G. Parisi, and F. Ritort, Phys. Rev. Lett. 74, 1012 (1995).

${ }^{34}$ F. G. Padilla and F. Ritort (unpublished).

${ }^{35}$ In the spherical SK model metastable states are absent. See A. Crisanti and H.-J. Sommers (unpublished).

${ }^{36}$ W. Krauth and M. Mézard (unpublished); W. Krauth and O. Pluchery, J. Phys. A 27, L715 (1994).

${ }^{37}$ G. Migliorini and F. Ritort, J. Phys. A 27, 7669 (1994).

${ }^{38}$ F. Ritort, Phys. Rev. Lett. 75, 1190 (1995); S. Franz and F. Ritort, Europhys. Lett. 31, 507 (1995); S. Franz and F. Ritort (unpublished).

${ }^{39}$ Y. Kuramoto, Chemical Oscillations, Waves and Turbulence (Springer, Berlin, 1984).

${ }^{40}$ J. W. Swift, S. H. Strogatz, and K. Wiesenfeld, Physica D 55, 239 (1992).

${ }^{41}$ T. Padmanabhan, Phys. Rep. 188, 285 (1990).

${ }^{42}$ L. L. Bonilla, J. A. Carrillo, and J. Soler, Phys. Lett. A 212, 55 (1996). 